\title{
EXPLOITING MULTI-TEMPORAL SENTINEL-1 SAR DATA FOR FLOOD EXTEND MAPPING
}

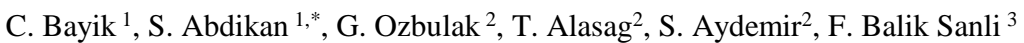 \\ ${ }^{1}$ BEU, Engineering Faculty, Dept. of Geomatics Engineering, 67100 Zonguldak, Turkey - (caglarbayik, sabdikan)@beun.edu.tr \\ 2 TUBITAK-BILGEM, 41470 Kocaeli, Turkey - (gokhan.ozbulak, tolga.alasag, said.aydemir)@ tubitak.gov.tr \\ ${ }^{3}$ YTU, Civil Engineering Faculty, Dept. of Geomatics Engineering, 34220 İstanbul, Turkey - fbalik@yildiz.edu.tr
}

\section{Commission ICWG III/IVa}

KEY WORDS: Flood, Disaster, Sentinel-1, Threshold, Change detection, Deep learning

\begin{abstract}
:
Recently, global climate change is one of the biggest challenges in the world. Dense downfall and following catastrophic floods are one of the most destructive natural hazards among all. Consequences do not only risk human life but also cause economical damage. It is critical rapid mapping of flooding for decision making and emergency services in river management. In this study, we apply a multi-temporal change detection analysis to investigate the flooded areas occurred in Edirne province of Turkey. The study area is located at the lower course of Meric River (Evros in Greece or Maritsa in Bulgarian) which is the border between Turkey and Greece. The river basin is dominated by cropland which suffers from strong catastrophic precipitation. This situation cause overflow of capacity of the dams located along the river and serious flooding occur. Due to its dynamic structure the region exposed to heavy flooding in the past. One of the biggest inundations was occurred at 2nd February 2015 which resulted severe devastation in both urban and rural areas. For the analyses of the temporal and spatial dynamics of the disaster we use Sentinel-1 Synthetic Aperture Radar (SAR) data due to its systematic frequent acquisition. A dataset of pre-event and post-event Sentinel-1 images within the January and February of 2015 period was acquired. Flooded areas were extracted with threshold, random forest and deep learning approaches.
\end{abstract}

\section{INTRODUCTION}

Freshwater resources cover less than $1 \%$ of Earth's surface (Harrison et al. 2016). As its nature it has a non-uniform spatial distribution which makes it more valuable. It provides natural environment for wildlife habitat and also source for human life such as agriculture, recreation and hydroelectric development. Flood disasters occur due to both climatological and anthropogenic reasons. Availability of near-real time inundation information is an important data for decision makers such as local government and insurance companies because floods effect both settlements and agriculture which cause economic losses (Tsyganskaya et al 2018).

Remote sensing and particularly synthetic aperture radar (SAR) sensors are suitable for data acquisition under dense precipitation conditions and provide rapid assessment and long term monitoring of the flooded areas. SAR system is sensitive to water due to specular reflection and able to acquire image both day and night which give it a characteristic specification. This provides utilization of SAR data for surface water and changes such as flood mapping is more common and feasible than optical data. Change detection approaches using SAR data includes backscatter intensity, polarimetry and interferometric coherence information for input and apply classification algorithms (Tsyganskaya et al 2018). Long et al (2014) applied thresholding technique on difference of SAR images and applied segmentation to extract flood extend using Envisat and Radarsat- 2 images. They also presented restriction of evaluation using optical data due to acquisition period and cloudiness of the data. Refice et al (2014) used coherence image in addition to intensity information of Cosmo-SkyMed data to detect areas affected by flood. A detailed review on flood inundation using SAR data could be found in Schumann et al (2015) and Tsyganskaya et al (2018).

In this study, the case of winter transboundary floods occurred in February 2015 over River Meric is under investigation in order to exploit multi-temporal S-1A data. The study area has been suffered of severe floods many times due to incapability of reservoir capacity and operational system of dams located on the river (Akkaya and Dogan 2016). Mallinis et al (2013) used single Envisat and two Landsat data, and applied object based image analysis for flood area mapping of flood occurred in February 2010 on Meric River. They concluded that Landsat result confirmed higher accuracy than Envisat result when comparing official extent map. Twele et al (2016) studied flooding occurred on Meric River in March 2015. An automatic thresholding and fuzzy logic based classification method was utilized to extract water and land classification using single post-event Sentinel-1 data, and Worldwiew-2 data for validation. They concluded the accuracy of VV is slightly better than $\mathrm{VH}$ result. A combination of dual polarimetric $\mathrm{S}-1$ and full polarimetric ALOS-2 imagery for flooded vegetation areas was investigated by Plank et al (2017). They applied different scenarios utilizing decomposition of S-1 and ALOS-2 data using unsupervised Wishart classification and an object-based post-classification refinement. It is concluded that fusion of two images increased the accuracy of mapping flooded vegetation area.

In this research the output of different flood mapping techniques are discussed applying threshold, random forest and deep learning approaches using backscatter intensity of S-1 dataset. The remaining sections are organized as; information 
given on study area and materials in section 2. Methodology is described in section 3. Spatial pattern of flood and maps are presented in section 4 . A summary of the preliminary output of the paper and further works are explained in section 5.

\section{TEST FIELD AND DATA SET}

\subsection{Test Field}

The study field is Meric River in Edirne Section (Evros in Greece or Maritsa in Bulgarian) which is in the northwest of Turkey. The field is also the natural border between Turkey and Greece (Figure 1). Meric is one of Balkan's greatest rivers. It springs in mountain of Bulgaria and flow through Greece and Turkey. Its length is 490 kilometers and its 172 kilometers are in Turkey. The main tributaries are Ergene, Arda and Tunca. Meric River in Edirne Section causes many serious floods that especially affects agricultural areas.

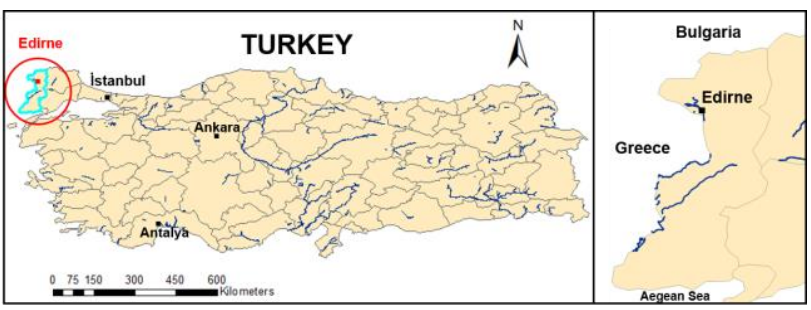

Figure 1. Meric River in Edirne Section

\subsection{Data Set}

For the analyses of flood, Sentinel-1A SAR data and RASAT optical images were used. New generation C-band SAR mission of Earth Space Agency (ESA) S-1 satellite provides large coverage of swath width $(250 \mathrm{~km})$, short repeat cycle $(6$ days with S-1A and S-1B), dual polarimetry mode (VV and VH), medium resolution $(10-20 \mathrm{~m})$ and narrow orbit tube for interferometric acquisitions. S-1A is acquired in Level-1 Single Look Complex (SLC) data format and is collected in the Interferometric Wide (IW) mode. These satellite features provide more ground information for the flood events. To extract to water bodies, VV polarization is used because it gives better results than VH polarization (Twele et al. 2016). Four images have same incidence angle $\left(33.7^{\circ}\right)$ and footprint. The footprint of images is shown in Figure 2.

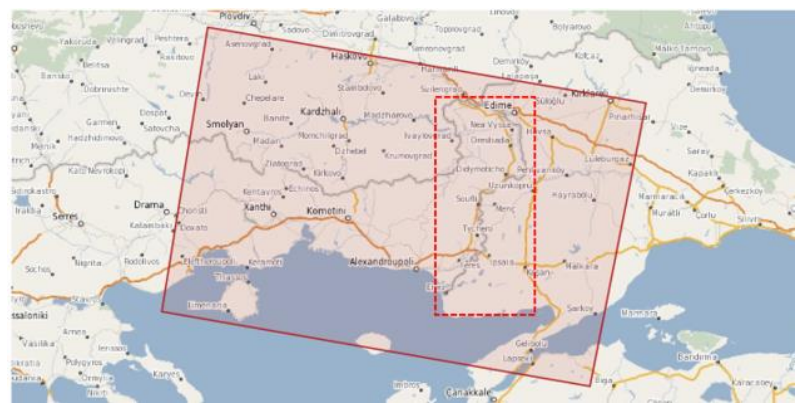

Figure 2. Footprint of Sentinel-1 images

In order to distinguish flood mapping, three Sentinel-1 images of pre-event and post-event within the January and February of 2015 period were acquired (Table 1). Temporal resolution of Sentinel-1 is 12 days.

RASAT is the second earth observation satellite of Turkey and was designed and assembled in TÜBİTAK (The Scientific and
Technological Research Council of Turkey). The characteristics of RASAT is given in Table 2. As Sentinel, RASAT images were also acquired pre-event and co-event.

\begin{tabular}{|c|c|c|c|}
\hline $\begin{array}{c}\text { Flood } \\
(02 / 02 / 2015)\end{array}$ & $\begin{array}{c}\text { Acquisition } \\
\text { Date }\end{array}$ & $\begin{array}{c}\text { Pass } \\
\text { Direction }\end{array}$ & Mode \\
\hline Pre-event & $17 / 01 / 2015$ & Descending & IW_SLC \\
\hline Post-event & $\begin{array}{c}10 / 02 / 2015 \\
22 / 02 / 2015\end{array}$ & $\begin{array}{c}\text { Descending } \\
\text { Descending }\end{array}$ & $\begin{array}{c}\text { IW_SLC } \\
\text { IW_SLC }\end{array}$ \\
\hline
\end{tabular}

Table 1. Sentinel-1 data.

\begin{tabular}{|l|c|}
\hline \multicolumn{1}{|c|}{ Characteristic } & Value \\
\hline Orbit type & Sun-synchronised \\
Orbital inclination & $98.1^{\circ}$ \\
Spatial resolution & $7.5 \mathrm{~m}$ (Panchromatic) \\
Radiometric resolution & $15 \mathrm{~m}$ (VNIR) \\
Temporal resolution & 8 bit \\
& 2.5 days \\
Spectral resolution & Pan: $0.42-0.73 \mu \mathrm{m}$ \\
& B3: Red: $0.58-0.73 \mu \mathrm{m}$ \\
& B2: Green: $0.55-0.58 \mu \mathrm{m}$ \\
& B1: Blue: $0.42-0.55 \mu \mathrm{m}$ \\
\hline
\end{tabular}

Table 2. Characteristics of RASAT.

\section{METHODOLOGIES}

In this study, some pre-processing have been applied to the Sentinel-1 images to extract the water bodies. Sentinel Application Platform (SNAP) software is used for these preprocesses that are Radiometric Calibration, Thermal Noise Removal, TOPSAR-Deburst, Multilook, Terrain Correction and Speckle filter. Three approaches were used to extract water bodies of Meric River. These are backscatter thresholding, random forest classification and deep learning classification approaches. The results obtained with these three methods are compared visually with the RASAT images $(7.5 \mathrm{~m} \times 7.5 \mathrm{~m})$ to figure out the detailed extraction of the flooded and nonflooded areas. Using pre and post event images changes of two periods between $17 / 01 / 2015$ and 10/02/2015 as first period, and $17 / 01 / 2015$ and 22/02/2015 as second period were analyzed using three methods. As shown in Figure 3 red color reflects the spatial extent of flooding water.

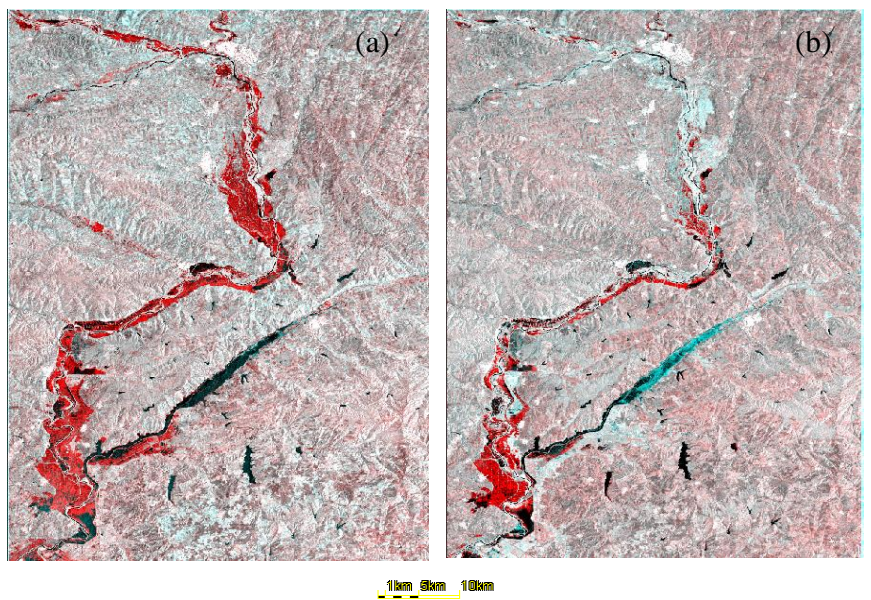

Figure 3. False color composition of flooding area; (a) RGB: $17 / 01 / 2015-10 / 02 / 2015-10 / 02 / 2015$, (b) RGB: 17/01/2015$22 / 02 / 2015-22 / 02 / 2015$ 


\subsection{Backscatter Thresholding}

Flood mapping with multi-temporal change detection analysis of radar data depends on radar backscatter. Backscatter results due to its reflectance value with water's surface. As a result of homogeneous and smooth surface of the water bodies, the return of radar pulses will be weak. Since return of radar pulse from dry areas will be stronger, characteristic can easily be determined. Water extraction with the help of threshold determined by backscatter value $\sigma^{0}(\mathrm{~dB})$ is a commonly used method. For each pixel of the SAR image, we determine the measured backscatter value, we can predict the probability of the pixel being a water structure. For this purpose, the histogram of backscatter value was analyzed. Low backscatter values correspond to the flooded areas and high backscatter values correspond to the non-flooded areas. First log-ratio image which shows the intensity difference was calculated and thresholding was applied for both periods (Cui et al. 2017).

\subsection{Random Forest Classification}

As an alternative to classical pixel and object-based classification methods, various learning-based algorithms are developed to obtain more accurate and reliable information from satellite images. One of them is Random Forest Classification (RFC) which is a community learning method and its algorithm is known as one of the most successful classification methods. The RFC method provides a unique predictive validity and model interpretability within known machine learning methods. RFC method provides better generalizations because of the random sampling and the improved properties of the techniques in community methods. For this reason, there are valid estimates (Horning, 2010).

\subsection{Deep Learning Classification}

The method proposed by Gong et al (2016) was applied onto the flood event in Edirne occurred in the early 2015. This method is based on a variant of the deep belief network called as Restricted Boltzmann Machines (RBM). In this approach, corresponding pixels from pre-event and post-event SAR images are vectorized in their local neighborhood and these vectors are concatenated as one vector for each pixel in interest. All concatenated vectors are then fed into RBM based deep belief network model with their labeling information for training. This model learns the change between local patches vectorized before with user provided labels. The labeling is marking the corresponding patches, which are not changing, as 1 and marking the changing patches as 0 .

The topology and the parameter setting of the RBM based change detection method is summarized in Table 3. The network has one input layer, three RBM layers and one output layer. The input layer, as explained above, takes the pixels in $5 \times 5$ neighborhood from pre-event and post-event SAR images as one concatenated vector. The first RBM layer is fed with this $2 \times 5 \times 5$ vector to project it into 500 -dimensional vector. The second RBM layer re-projects the 500-dimensional vector into 250-dimensional vector. Similarly, the second RBM layer projects 250-dimensional vector into 200-dimensional vector. The output layer then classifies the 200-dimensional feature vector as 1 (no change case) or 0 (change case) by using negative log likelihood as loss function. The conjugate gradient is used as optimization algorithm with a learning rate of 0.005 and a momentum of 0.9 . The number of epoch is 10 for training.

\begin{tabular}{|l|l|}
\hline Parameters & Setting \\
\hline Learning Rate & 0.005 \\
Updater & Nesterov \\
Momentum & 0.9 \\
Optimization & Conjugate Gradient \\
Regularization & Yes \\
Topology & $25-500-250-200-2$ \\
Weight Initialization & Xavier \\
RBM Loss Function & XENT \\
Softmax Loss Function & Negative Log Likelihood \\
\hline
\end{tabular}

Table 3. The properties of RBM based change detection method.

\section{RESULTS AND DISCUSSIONS}

The analysis conducted to extract water surface and its spatial extent. In all approaches the results provide us two classes as water which shows the inundation area and others where did not effected by flooding between SAR image acquisitions.

First analyze is conducted using histogram of backscatter values and threshold values are determined (Figure 3). The threshold values were set as $-15.47 \mathrm{~dB},-15.59 \mathrm{~dB}$ and $-17.46 \mathrm{~dB}$ in date order. After determining the backscatter threshold, a binary image was created. As seen in Figure 4 flooded areas are represented with black and non-flooded areas with white color (Figure $4 \mathrm{a}$ and $4 \mathrm{~d}$ ). In the first period the extention of river flooding is much more than the second period. The extention is reduced within following twelve days.

In the analysis of RFC two parameters should be defined by the user to initiate the algorithm. These parameters are number of training samples and number of trees to be improved to determine the best partition (Breiman and Cutler, 2005). In this study, classifications were carried out by taking number of training samples as 5000 and number of trees as 10 . The results of the classification for both data pair is shown in Figure $4 \mathrm{~b}$ and $4 \mathrm{e}$. In the results RFC performed smoother output comparing to other results. There are less distributed water bodies in both periods.

In the results of RBM approach the flood delineated areas showed similar spatial distribution with other two approaches. In the results it is indicated that apart from river sides randomly distributed water bodies are also extracted (Figure $4 \mathrm{c}$ and $4 \mathrm{e}$ ). These regions are covered with cropland and backscatter values of cropland showed similar values as water due to high moisture content.

For the evaluation of the results higher resolution optical images are acquired and changes has been visualized as shown in Figure 4. However, due to hydrological dynamics of the river the water surface extend changes rapidly and the lack of cloudfree data did not let to us to acquire better optical data and validate the results. Even this condition RASAT image gave preliminary results of the extension of the flooding at the northern part of the study area (Figure 4 and 5). RASAT images clearly showed that inundation covered agricultural areas along the river and cause damage over croplands. 


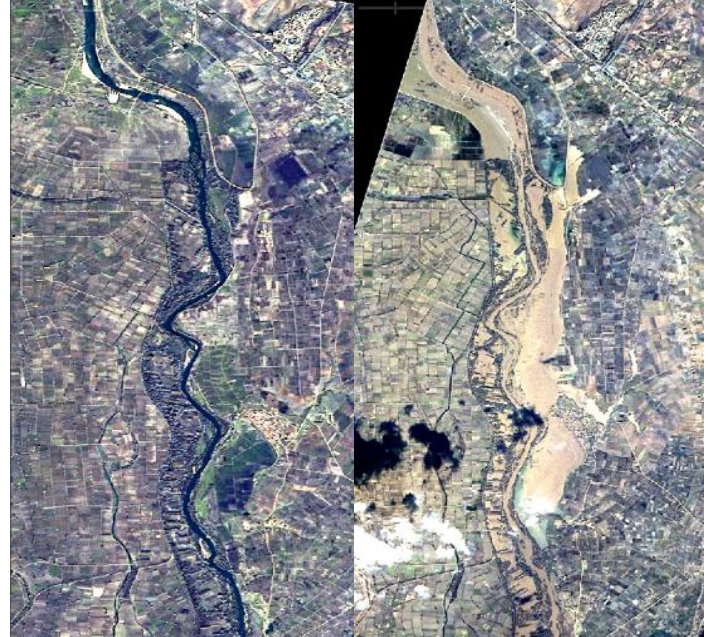

Figure 4. Pre and co-event of RASAT images; left is 2013.12.03 and right is 2015.02.02

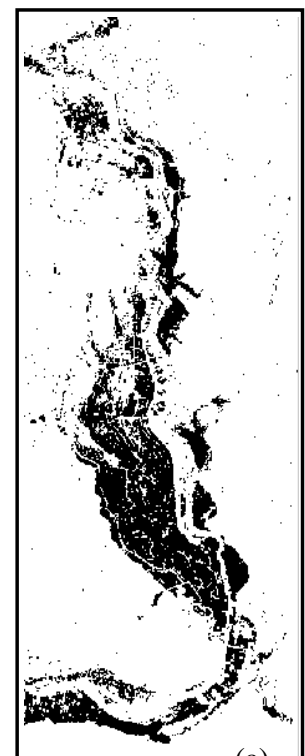

(a)

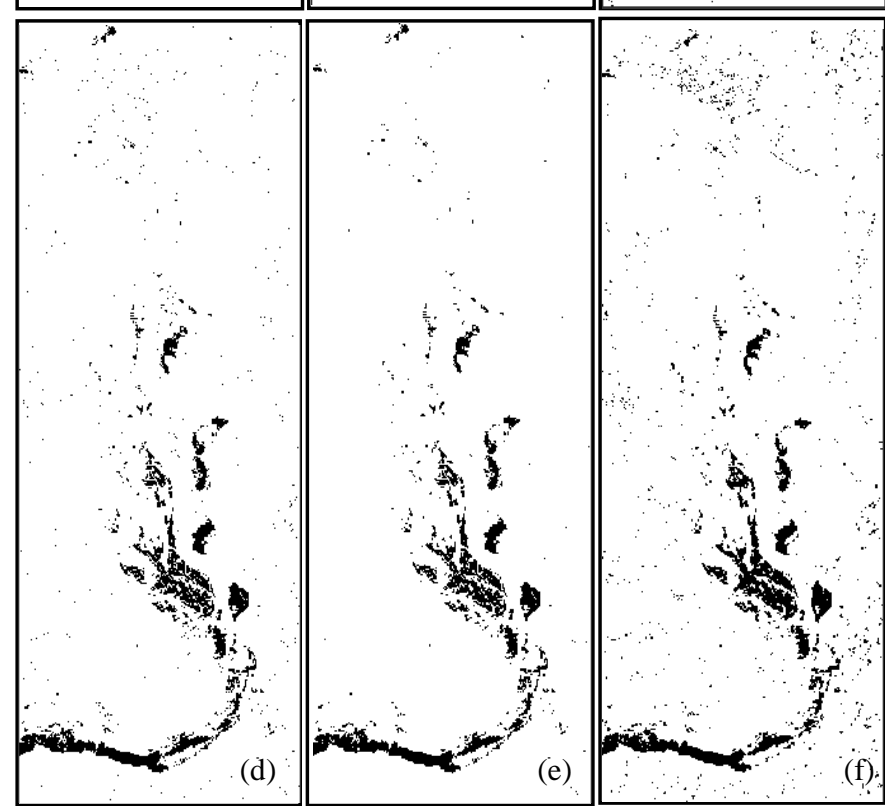

Figure 5. Results of change detection; first period (17.01-10.02) (a) thresholding, (b) RFC, (c) RBM and second period (17.0122.02) (d) thresholding, (e) RFC, (f) RBM

Using three methods six results are evaluated according to multi-temporal analysis and the water extent of inundation is indicated in Table 5. The results of RFC method gave smaller amount of water extent against other methods in both time periods. The highest spatial extent of flooding is extracted with RBM method in both time periods (Table 4).

Accuracy assessment analysis is applied for each change detection result which are presented in Figure 4 and embed in Table 5. For the accuracy analysis 300 pixels are distributed randomly on SAR images and accuracies are estimated. All results performed high overall accuracies which are more than $90 \%$. Even log-ratio and thresholding followed approach is a simple method it provides good results which makes it practical for the monitoring of rapid flooding for large areas. Although, the results of RFC and RBM are close to each other RFC approach provides higher accuracies in both periods which makes it prominent approach in this case study.

\begin{tabular}{|c|cc|}
\hline & \multicolumn{2}{|c|}{ Thresholding } \\
& $\mathbf{1 7 . 0 1 - 1 0 . 0 2}$ & $\mathbf{1 7 . 0 1 - 2 2 . 0 2}$ \\
\hline Water $\left(\mathbf{k m}^{\mathbf{2}}\right)$ & 115.75 & 32.89 \\
Others $\left(\mathbf{k m}^{2}\right)$ & 642.61 & 725.48 \\
& \multicolumn{2}{|c|}{} \\
\hline & Random Forest & Classification \\
& $\mathbf{1 7 . 0 1 - 1 0 . 0 2}$ & $\mathbf{1 7 . 0 1 - 2 2 . 0 2}$ \\
Water $\left(\mathbf{k m}^{\mathbf{2}}\right)$ & 86.04 & 31.25 \\
Others $\left(\mathbf{k m}^{2}\right)$ & 672.32 & 727.12 \\
& \multicolumn{2}{|c}{} \\
& Deep Learning & Classification \\
& 17.01-10.02 & $\mathbf{1 7 . 0 1 - 2 2 . 0 2}$ \\
Water $\left(\mathbf{k m}^{\mathbf{2}}\right)$ & 132.45 & 40.14 \\
Others $\left(\mathbf{k m}^{2}\right)$ & 625.92 & 718.22 \\
\hline
\end{tabular}

Table 4. Water surface extent of flooding

\begin{tabular}{|c|c|c|}
\hline \multirow{4}{*}{$\begin{array}{c}\text { Overall } \\
\text { accuracy } \\
\text { Kappa }\end{array}$} & \multicolumn{2}{|c|}{ Thresholding } \\
\hline & 17.01-10.02 & 17.01-22.02 \\
\hline & $94.6 \%$ & $97.6 \%$ \\
\hline & $83.1 \%$ & $80.9 \%$ \\
\hline \multirow{4}{*}{$\begin{array}{c}\text { Overall } \\
\text { accuracy } \\
\text { Kappa }\end{array}$} & \multicolumn{2}{|c|}{ Random Forest Classification } \\
\hline & 17.01-10.02 & 17.01-22.02 \\
\hline & $96.3 \%$ & $99 \%$ \\
\hline & $84.6 \%$ & $90.9 \%$ \\
\hline \multirow{4}{*}{$\begin{array}{c}\text { Overall } \\
\text { accuracy } \\
\text { Kappa }\end{array}$} & \multicolumn{2}{|c|}{ Deep Learning Classification } \\
\hline & 17.01-10.02 & 17.01-22.02 \\
\hline & $95 \%$ & $98.6 \%$ \\
\hline & $79.9 \%$ & $89.3 \%$ \\
\hline
\end{tabular}

Table 5. Accuracy assessment of change detection results 


\section{CONCLUSIONS}

Continuous monitoring of Meric River contributes to its protection and management of transboundary floods for mitigation of possible risks. Particularly agricultural fields are under risk and possible continuous damage may cause lasting socio-economic problems in this region. In this study, spatial and temporal analysis of flooding which occurred at the border of Turkey and Greece is investigated. To this aim, S-1 SAR images are utilized and inundation extend is extracted using three methods. All three methods provided sufficient outputs while RFC indicated slightly better results in both time periods.

As a further research a longer time period of S-1 dataset will be evaluated to determine the seasonal dynamics of the flooding using digital elevation model. The contribution of coherence image and texture information will be also investigated to improve the accuracy and map reliable monitoring.

\section{ACKNOWLEDGEMENTS}

Deep learning based experiments were performed in Aydes Uzal product that is developed by TUBITAK BILGEM for Republic of Turkey Prime Ministry Disaster and Emergency Management Presidency (AFAD) (Project No: B740-100289).

\section{REFERENCES}

Akkaya, U., Doğan, E., 2016. Generation of 2D flood inundation maps of Meriç and Tunca Rivers passing through Edirne city center. Geofizika 33, 15-34.

Breiman L.,Cutler A., 2005. Random forest, http://www.stat.berkeley.edu/ breiman/RandomForests/cc_hom e.htm, *Last visit: 10 February 2018].

Cui, B., Zhang, Y., Yan, L., Cai, X., 2017. A SAR Intensity Images Change Detection Method Based on Fusion Difference Detector and Statistical Properties. ISPRS Annals of the Photogrammetry, Remote Sensing and Spatial Information Sciences 4, 439.

Gong, M., Zhao, J., Liu, J., Miao, Q., Jiao, L., 2016. Change detection in synthetic aperture radar images based on deep neural networks. IEEE Transactions on Neural Networks and Learning Systems 27, 125-138.

Harrison, I.J., Green, P.A., Farrell, T.A., Juffe-Bignoli, D., Sáenz, L., Vörösmarty, C.J., 2016. Protected areas and freshwater provisioning: a global assessment of freshwater provision, threats and management strategies to support human water security. Aquatic Conservation: Marine and Freshwater Ecosystems 26, 103-120.

Horning, N., 2010. Random Forests: An algorithm for image classification and generation of continuous fields data sets, Proceedings of the International Conference on Geoinformatics for Spatial Infrastructure Development in Earth and Allied Sciences, Osaka, Japan.
Long, S., Fatoyinbo, T.E., Policelli, F., 2014. Flood extent mapping for Namibia using change detection and thresholding with SAR. Environmental Research Letters 9, 035002.

Mallinis, G., Gitas, I.Z., Giannakopoulos, V., Maris, F., TsakiriStrati, M., 2013. An object-based approach for flood area delineation in a transboundary area using ENVISAT ASAR and LANDSAT TM data. International Journal of Digital Earth 6, 124-136.

Plank, S., Jüssi, M., Martinis, S., Twele, A., 2017. Mapping of flooded vegetation by means of polarimetric Sentinel-1 and ALOS-2/PALSAR-2 imagery. International Journal of Remote Sensing 38, 3831-3850.

Refice, A., Capolongo, D., Pasquariello, G., D'Addabbo, A., Bovenga, F., Nutricato, R., Lovergine, F.P., Pietranera, L., 2014. SAR and InSAR for flood monitoring: Examples with COSMO-SkyMed data. IEEE Journal of Selected Topics in Applied Earth Observations and Remote Sensing 7, 2711-2722.

Schumann, G.J.-P., Moller, D.K., 2015. Microwave remote sensing of flood inundation. Physics and Chemistry of the Earth, Parts A/B/C 83, 84-95.

Tsyganskaya, V., Martinis, S., Marzahn, P., Ludwig, R., 2018. SAR-based detection of flooded vegetation-a review of characteristics and approaches. International Journal of Remote Sensing 39, 2255-2293.

Twele, A., Cao, W., Plank, S., Martinis, S., 2016. Sentinel-1based flood mapping: a fully automated processing chain. International Journal of Remote Sensing 37, 2990-3004 